\title{
A Geophysical Model Function for Wind Speed Retrieval from C-Band HH-polarized Synthetic Aperture Radar
}

\author{
Zhang Biao ${ }^{1,{ }^{*}}$, Mouche Alexis ${ }^{2}$, Lu Yiru ${ }^{1}$, Perrie William ${ }^{3}$, Zhang Guosheng ${ }^{1}$, Wang He 4
}

1 School of Marine Sciences, Nanjing University of Information Science and Technology, Nanjing 210044, China

2 IFREMER, Univ. Brest, CNRS, IRD, Laboratoire d'Océanographie Physique et Spatiale (LOPS), Brest, France

${ }^{3}$ Bedford Institute of Oceanography, Fisheriesand Oceans Canada, Dartmouth, NS B2Y 4A2, Canada

${ }^{4}$ National Ocean Technology Center, State Oceanic Administration, Tianjing, China

* Corresponding author : Biao Zhang, email address : zhangbiao@nuist.edu.cn ;

alexis.mouche@ifremer.fr ; lyr@nuist.edu.cn ; william.perrie@dfo-mpo.gc.ca ; zgsheng001@gmail.com

\begin{abstract}
:
Synthetic aperture radar (SAR) imagery is routinely acquired at $\mathrm{HH}$-polarization in high latitude areas for measuring surface wind over the ocean. However, in the contrary of VV-polarization, there is no $\mathrm{HH}$ polarization geophysical model function (GMF) exists to directly retrieve wind speed from SAR images. In general, HH-polarized Normalized Radar Cross Section (NRCS) is thus converted into VV-polarization and then conventional CMOD functions are used with auxiliary wind direction information for wind speed retrieval. In this letter, we propose a new GMF for SAR ocean surface wind speed retrieval, called $\mathrm{CMODH}$, which relates the C-band NRCS acquired at $\mathrm{HH}$-polarization over the ocean, to the 10-m height wind speed, incident angle and relative wind direction. We first use more than 220,000 ENVISAT ASAR radar backscatter measurements collocated with ASCAT winds to derive the CMODH coefficients. Subsequently, 1459 RADARSAT-2 (RS-2) and 428 Sentinel-1A/B (SI-1A/B) HH-polarized SAR acquisitions under different wind speeds are matched to in situ buoy observations to validate CMODH. The statistical comparisons between SAR-observed and simulated NRCS show a bias of $-0.07 \mathrm{~dB}$ and a root mean square error of $1.62 \mathrm{~dB}$ for RS-2, and $-0.01 \mathrm{~dB}$ and $2.48 \mathrm{~dB}$ for $\mathrm{S} 1-1 \mathrm{~A} / \mathrm{B}$. These results suggest that the proposed $\mathrm{CMODH}$ has the potential to directly retrieve ocean surface wind speeds using C-band SAR images acquired at $\mathrm{HH}$-polarization, with no need for NRCS transformation by using various empirical and theoretical polarization ratio models.
\end{abstract}

Keywords : HH-polarization, ocean surface wind speed, synthetic aperture radar (SAR) 
YNTHETIC aperture radar (SAR) is a very useful $\checkmark$ microwave sensor for remote sensing of ocean surface features with ability for high-resolution and almost all-weather observations including night and day. Spaceborne SARs are on onboard polar-orbiting satellites and thus have the capability to observe high-latitude areas with shorter revisit time than middle-and low-latitude regions. In particular, they are routinely used to monitor seasonally or annual variations in seaice in the Arctic, Antarctic, and other high-latitude areas such as Chukchi Sea, the Labrador Sea, or the Beaufort Sea. When only one co-polarized channel is available, HH-polarization is preferred for sea-ice application because the contrast with ocean is more pronounced than at VV-polarization [1]. As a consequence, the acquisition strategy at high latitudes gives priority to $\mathrm{HH}$-polarization (and cross-polarization when available). In the contrary, $\mathrm{VV}$ is widely at mid-latitude for ocean applications, including wind field measurements.

Moreover, the high-resolution ocean surface wind is not only a key complementary geophysical parameter to investigate sea-ice motion and melt but also the coupling between ocean and the marginal ice zone (MIZ) in high-latitude regions. Long time-series satellite-tracked sea-ice motion observations reveal that changes in wind speed are linked to the observed increase in ice drift speeds in the Center Arctic, on climate timescales [2]. Antarctic sea-ice motions observed at large scale with satellites also suggest that significant trends in Antarctic ice drift can be statistically related to local winds in most geographic areas [3]. For example, in coastal East Antarctica, persistent katabatic winds from the ice sheet's interior cause widespread snow erosion and enhance the melt of blue ice and firn snow [4].

SAR ocean surface wind speed retrieval is usually based on geophysical model functions (GMFs) [5]-[7], which are derived from VV-polarized scatterometer observations. Conventional scatterometer-derived GMFs have been demonstrated to be applicable for moderate wind speed retrieval with an accuracy of $1.5 \mathrm{~m} / \mathrm{s}$ from ERS, ENVISAT ASAR, Sentinel-1A, or RADARSAT-2 VV-polarized SAR images [8]-[12]. Mouche and Chapron [13] pioneered the idea of relying directly and massively on SAR data to describe the relationship between NRCS and ocean surface wind in both co-polarizations and possibly derive GMF from SAR data. 
Recently, a new GMF has been proposed for C-band SAR coastal wind speed mapping [14], by using SAR measurements acquired in VV-polarization collocated with buoy observations. However, no similar well-documented wind retrieval model exists to derive wind speed from the HH-polarized SAR imagery. Therefore, various empirical and theoretical polarization ratio (PR) models have been developed to transform normalized radar cross section (NRCS) from $\mathrm{HH}$ into VV-polarization [15]-[20], along with CMOD GMFs to retrieve wind speed. A systematic comparison of PR models for ocean surface wind retrieval using ENVISAT ASAR images shows a small wind speed underestimation or overestimation at very small or large incident angles, respectively [21].

Although the CMOD GMF-PR approach constitutes a practical hybrid model for wind speed retrieval from SAR images acquired at HH-polarization, the NRCS transformation certainly induces error in wind speed estimation. In order to directly retrieve wind speeds using $\mathrm{HH}$-polarized SAR imagery, we develop a new GMF relating NRCS at $\mathrm{HH}$-polarization to radar incident angle, wind speed at $10 \mathrm{~m}$ height, and relative wind direction. Finally, to complement the VV-polarization, the next European scatterometer mission onboard MetOp-SG will also operate at HH-polarization (and VH-polarization) [22]. The proposed GMF should, thus, directly benefit to this forthcoming satellite mission. This letter is organized as follows. Data sets are described in Section II. GMF development and validation are presented in Section III. Summary and conclusion are given in Section IV.

\section{DATA SET}

In this letter, more than 2700 ENVISAT/ASAR images acquired in wide swath mode (WSM) in 2009 are collocated with MetOp/ASCAT and ECMWF winds to develop the HH-polarized GMF. The incident angles of WSM are between $16^{\circ}$ and $42^{\circ}$ in the near and far ranges, respectively. SAR acquisitions in WSM cover about $400 \mathrm{~km}$ in the range direction and can be more than $1000 \mathrm{~km}$ in the azimuth direction. We processed all ASAR WSM products to derive the NRCS, incident angles, and azimuth angles at a spatial resolution cell of $12.5 \mathrm{~km}$. These measurements are then matched to ASCAT wind speeds and directions. This results in more than 220000 collocations. The ENVISAT ASAR and ASCAT orbits and field of view enable temporal intervals of less than $1 \mathrm{~h}$, for collocation. This collocated data set has already been used to analyze wind sensitivity for different incident angles and azimuth angles [13].

In order to validate the proposed GMF, we collect 1352 RS-2 SAR images acquired in quad-polarization $(\mathrm{HH}+\mathrm{HV}+$ $\mathrm{VH}+\mathrm{VV})$ and 107 dual-polarization $(\mathrm{HH}+\mathrm{HV})$ imaging modes between October 2008 and April 2013, and 428 Sentinel-1A/B (Sl-1A/B) images acquired in extra wide swath (EW) mode between October 2014 and April 2018. A summary of the parameters for the RS-2 quad-and dualpolarization modes, including incident angles, spatial resolutions, swaths, and noise-equivalent sigma-zero (NESZ) values, is given in Table I. The EW mode of S1-1A/B data has the capability to observe the ocean surface with dual-polarization $(\mathrm{HH}+\mathrm{HV})$ channels. Table II contains the major parameters
TABLE I

MAJOR PARAMETERS OF THE RS-2 QUAD-AND DUAL-POLARIZATION IMAGING MODES

\begin{tabular}{lcc}
\hline Parameter & quad-polarization mode & $\begin{array}{c}\text { dual-polarization } \\
\text { ScanSAR Narrow mode }\end{array}$ \\
\hline Polarization & $\mathrm{HH}+\mathrm{HV}+\mathrm{VH}+\mathrm{VV}$ & $\mathrm{VV}+\mathrm{VH}$ \\
Incident angles & $20^{\circ}-49^{\circ}$ & $20^{\circ}-46^{\circ}$ \\
Azimuth resolution & $8 \mathrm{~m}$ & $60 \mathrm{~m}$ \\
Range resolution & $5.4 \mathrm{~m}$ & $79.9-37.7 \mathrm{~m}$ \\
Swath & $25 \mathrm{~km}$ & $300 \mathrm{~km}$ \\
NESZ & $-36.5 \pm 3 \mathrm{~dB}$ & $28.5 \pm 2.5 \mathrm{~dB}$ \\
\hline
\end{tabular}

TABLE II

Major Parameters of THE SL-1A/B IW AND EW IMAgIng ModeS

\begin{tabular}{lc}
\hline Parameter & Extra wide swath (EW) arrow mode \\
\hline Polarization & $\mathrm{HH}+\mathrm{HV}$ \\
Incident angles & $19^{\circ}-47^{\circ}$ \\
Azimuth resolution & $40 \mathrm{~m}$ \\
Range resolution & $20 \mathrm{~m}$ \\
Swath & $400 \mathrm{~km}$ \\
NESZ & $-22 \mathrm{~dB}$ \\
\hline
\end{tabular}

for EW mode. All RS-2 and Sl-1A/B SAR images are collocated with 102 in situ National Data Buoy Center (NDBC) buoys in the Gulf of Alaska, off the East and West coasts of the USA, and the European Seas. The time interval for the collocation is smaller than $30 \mathrm{~min}$. We process the NRCS at $\mathrm{HH}$-polarization at a spatial resolution of $10 \mathrm{~km}$ to match the resolution used by Mouche and Chapron [13], where medium (instead of high) resolution was used to be in line with collocated wind field from ASCAT. This approach results in 1527 and 461 collocated data pairs for RS-2 and Sl-1A/B, respectively, which are used to compare simulated and SAR-measured NRCS.

\section{Model Development And Validation}

Similar to NRCS at VV-polarization, HH-polarized NRCS also depend on radar incident angle, wind speed, and direction (see [13, Fig. 3(b) and (d)]). Therefore, we describe NRCS at HH-polarization by a nonlinear mapping function of the incident angle, wind speed, and relative wind direction (the angle between the true wind direction and the radar observation direction), which is given as

$$
\begin{aligned}
\sigma_{0}^{\mathrm{hh}}(v, \phi, \theta)=\left(B_{0}(v, \theta)[1+\right. & B_{1}(v, \theta) \cos (\phi) \\
& \left.\left.+B_{2}(v, \theta) \cos (2 \phi)\right]\right)^{p}
\end{aligned}
$$

where the isotropic $B_{0}$, the upwind/downwind $B_{1}$, and the upwind/crosswind $B_{2}$ amplitude terms are the functions of wind speed $v$ and incident angle $\theta$. The superscript $p$ is a constant with a value of 1.6. The superscript $p$ and the transfer functions used to define $B_{0}, B_{1}$, and $B_{2}$ are adopted from CMOD5 [6] for use in this study. The proposed form of the GMF is almost the same as that of CMOD5 excepted for the $p$ exponent, on $B_{0}$.

For each incident angle and wind speed bin, using the collocated ENVISAT ASAR and ASCAT data set, we first analyze the NRCS at HH-polarization as a function of azimuth angle 


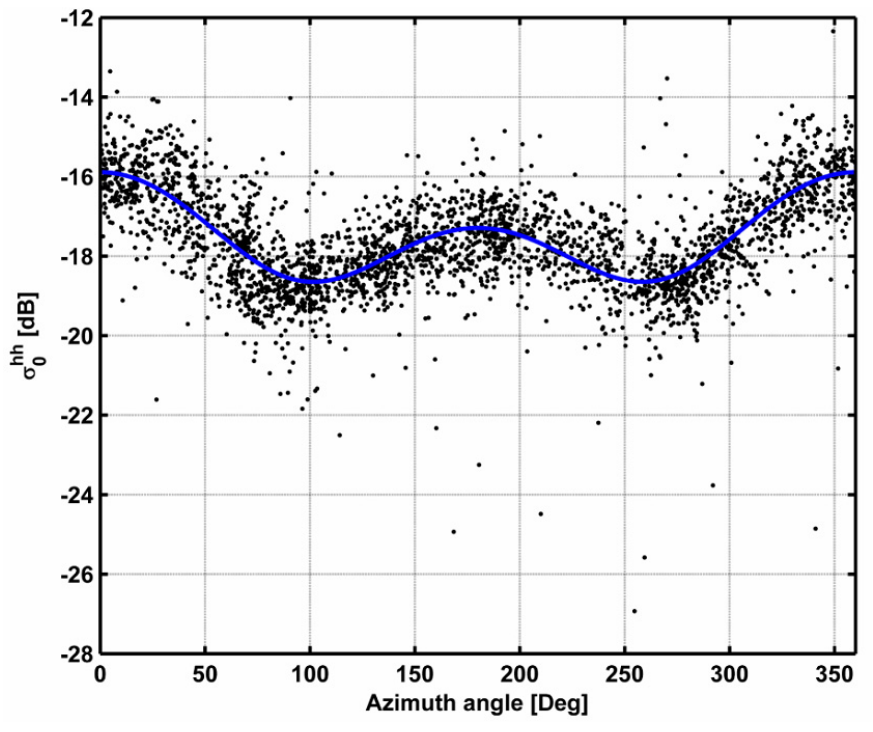

Fig. 1. HH-polarized NRCS as a function of azimuth angle, for a $7 \mathrm{~m} / \mathrm{s}$ wind speed and $35^{\circ}$ incidence angle. Black dots are SAR-observed NRCS at $\mathrm{HH}$-polarization. Blue line represents regression results.
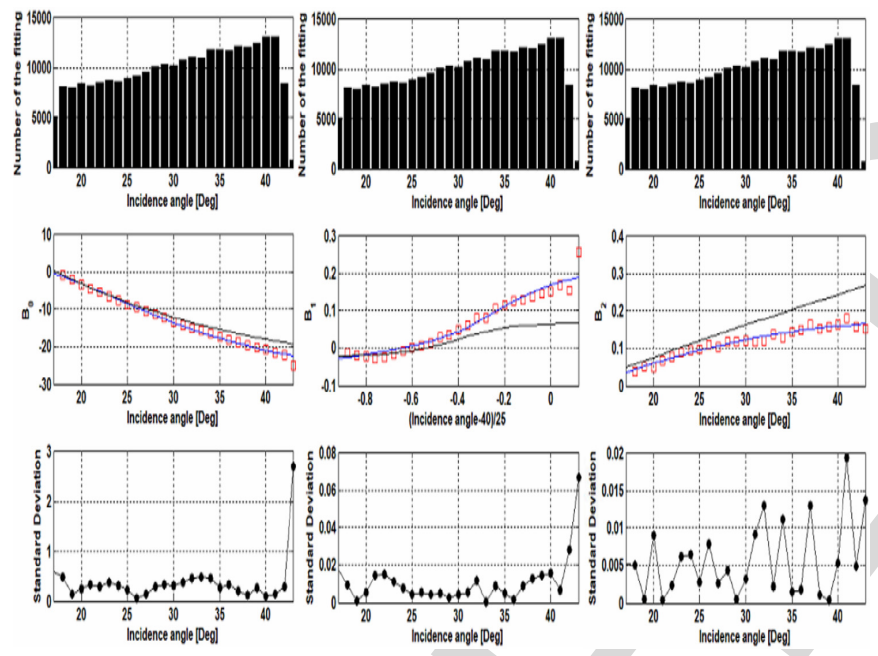

Fig. 2. Number of the data for (Top) $B_{i}$ coefficients derivation, (Middle) $B_{i}$ coefficients, and (Bottom) standard deviation of the regression as a function of incident angle for $7 \mathrm{~m} / \mathrm{s}$ wind speed. $B_{i}$ coefficients derived from (1), the regression analysis approach, and the CMOD5 are represent as red squares, and blue and black solid lines, respectively.

to determine the corresponding $B_{i}(i=0,1,2)$ coefficients by using a regression analysis approach. Fig. 1 presents the ASAR-observed NRCS (black dots) versus azimuth angles for $7 \mathrm{~m} / \mathrm{s}$ wind speed and $35^{\circ}$ incident angle. Fig. 1 clearly shows the azimuth modulation of NRCS at HH-polarization. Estimated NRCS values using (1) are indicated in the blue solid line in Fig. 1, which are in good agreement with the radar observations, suggesting that (1) is appropriate to describe the proposed GMF.

Subsequently, to further assess the rationality of the functional form, $B_{i}$ coefficients are also analyzed as a function of wind speed and incident angle. The top three panels in Fig. 2 show the number of data used for $B_{i}$ coefficients derivation. For $7 \mathrm{~m} / \mathrm{s}$ wind speed and different incident angles, $B_{i}$ coefficients derived from (1), the regression analysis

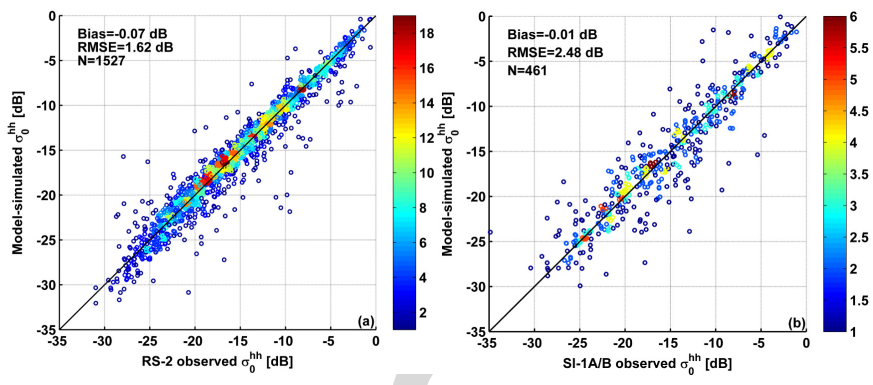

Fig. 3. Comparisons of observed NRCS in HH-polarization ( $x$-axis) from (a) RS-2 and (b) S1-A/B with CMODH model simulations ( $y$-axis). The color bar indicates the scatter density.

approach, and the CMOD5 function are represented as red squares, and blue and black solid lines, in the middle three panels of Fig. 2.

Overall, the trend of $B_{i}$ coefficients obtained by our analysis and those provided by CMOD5 GMF are in very good agreement excepted at the largest incident angle of $43^{\circ}$. The $B_{0}$ values from the regression analysis and from CMOD5 estimation are almost identical at low incident angles $\left(<23^{\circ}\right)$. As incident angles increase, the discrepancies between $B_{i}$ coefficients obtained for $\mathrm{HH}$ from our regression analysis and from CMOD5 gradually increase. This is in line with a known different sensitivity to ocean sea-surface backscattering between VV- and HH-polarizations. Indeed, at C-band, polarization effect is strongly incident angle-dependent. In particular, our analysis confirms that $B_{1}$ values at $\mathrm{VV}$ are smaller than at $\mathrm{HH}$, while $B_{0}$ and $B_{2}$, are larger at VV.

The standard deviations of the regression analysis for each incident angle bin are illustrated in the bottom three panels in Fig. 2. The standard deviation for the large incident angle bin of $43^{\circ}$ is much larger than those for other incident angle bins, which is possibly caused by the very few data numbers in this bin. Consequently, we do not use NRCS for this large incident angle bin to develop our $\mathrm{HH}$-polarized GMF. In this study, $B_{i}$ coefficients are determined via a nonlinear weighted regression approach by minimizing the standard deviation between ENVISAT ASAR observations and regression analysis for all wind speeds and incident angle bins. The number of the data in each wind speed and incident angle bin is considered as a weighted factor for the regression analysis. The final model formulation and 28 coefficients for HH-polarization are given in the Appendix. Since our collocated data sets both involve NRCS at HH- and VVpolarization, we also use (1) to derive the coefficients of GMF for VV-polarization, which are shown in the Appendix. Therefore, one can estimate NRCS at HH-and VV-polarization using (1) and coefficients with given incident angle, wind speed, and direction.

To assess the accuracy of the proposed GMF, we make statistical validations using collocated RS-2 and Sl-1A/B observations and buoy measurements. Fig. 3(a) shows the simulated and SAR-measured NRCS at HH-polarization, with a bias of $-0.07 \mathrm{~dB}$ and a RMSE of $1.62 \mathrm{~dB}$. For comparison between model simulations and S1-A/B observations, the bias and RMSE are -0.01 and $2.48 \mathrm{~dB}$, which are shown in Fig. 3(b). For NRCS comparisons, the RS-2 data have smaller 
RMSE than the S1-1A/B data. The reason for this result may be associated with two factors: 1) RS-2 has lower NESZ than the $\mathrm{S} 1-1 \mathrm{~A} / \mathrm{B}$ and 2) the collocations between $\mathrm{S} 1-1 \mathrm{~A} / \mathrm{B}$ and buoy data are less accurate than that of RS-2. For S1-1A/B collocation, some buoys are outside the SAR image which leads to the large spatial interval of the collocation. However, for RS-2 collocation, each buoy locates in the SAR image and thus there is almost no spatial match error.

\section{CONCLUSION}

Accurate wind field information in high latitudes and polar regions plays an important role in sea-ice motion and melt simulations and predictions. The high sensitivity of SAR backscatter at HH-polarization to sea-ice makes it more preferable for sea-ice monitoring, rather than VV-polarization. However, unlike CMOD GMFs, no HH-polarized GMF can be directly used to retrieve ocean surface wind speed. As a result, various empirical and theoretical PR models are used to transform NRCS from HH into VV-polarization, which induces inevitable errors in the retrieved wind speeds. In order to solve this problem, we develop a new HH-polarized GMF for C-band SAR, taking advantage of a large number of C-band SAR data and collocated scatterometer wind measurements. The proposed GMF has potential to improve SAR wind speed retrieval accuracy and can be used to directly retrieve wind speed in mixing areas of ice and water, like the MIZ.

The new GMF relates NRCS at HH-polarization to radar incident angle, wind speed at $10 \mathrm{~m}$ height, and relative wind direction. We use a nonlinear weighted regression method and the collocated data set (ENVISAT ASAR backscatter and ASCAT winds) to derive all the coefficients in the GMF. Furthermore, we use an independent matching of data (RS-2 and S1-1A/B backscatter and buoy measurements) to validate the proposed GMF. The statistical comparisons between radar observations and GMF simulations show a bias of $-0.07 \mathrm{~dB}$ and a root-mean-square error of $1.62 \mathrm{~dB}$ for RS-2, and $-0.01 \mathrm{~dB}$ and $2.48 \mathrm{~dB}$ for S1-1A/B, respectively. Based on this new GMF, we do not need to make a NRCS transformation with a PR model before wind speed retrieval. The scatterometer (SCA) onboard EUMETSAT Polar System-Second Generation (EPS-SG) mission shall provide observations with VV-, HH-, or VH-polarization. The addition of HH- or VH-polarization is considered as an option for extending the upper limit of the dynamic measurement range. Therefore, the proposed GMF can be a benefit for EPS-SG SCA wind retrieval. Moreover, this also opens new perspectives for the forthcoming launch of the RADARSAT Constellation Mission (RCM) with new polarization configurations.

\section{APPENDIX \\ CMODH MODEL FoRMULATION AND COEFFICIENTS}

The form of the CMODH model is

$$
\begin{aligned}
\sigma_{0}^{\mathrm{hh}}(v, \phi, \theta)=\left(B_{0}(v, \theta)[1\right. & +B_{1}(v, \theta) \cos (\phi) \\
& \left.\left.+B_{2}(v, \theta) \cos (2 \phi)\right]\right)^{p}
\end{aligned}
$$

where $p$ is constant with a value of $1.6, B_{0}, B_{1}$, and $B_{2}$ are functions of wind speeds $v$ and the incidence
TABLE III

\begin{tabular}{|c|c|c|c|}
\hline Function & Coefficients & $\mathrm{HH}$ & VV \\
\hline \multirow[t]{18}{*}{$B_{0}$} & $c_{1}$ & -0.72722756511 & -0.13393789593 \\
\hline & $c_{2}$ & -1.1901195406 & -0.74081314533 \\
\hline & $c_{3}$ & 0.33968637656 & 0.34811480603 \\
\hline & $c_{4}$ & 0.086759069544 & 0.019382338942 \\
\hline & $c_{5}$ & 0.003090124916 & -0.008066293463 \\
\hline & $c_{6}$ & 0.011761378188 & 0.006426074015 \\
\hline & $c_{7}$ & 0.129158495658 & 0.096343783534 \\
\hline & $c_{8}$ & 0.083506931034 & 0.042280179737 \\
\hline & $c_{9}$ & 4.092557781322 & 5.007750349297 \\
\hline & $c_{10}$ & 1.211169044551 & 0.717396068916 \\
\hline & $c_{11}$ & -1.119776245438 & -1.501296438845 \\
\hline & $c_{12}$ & 0.579066509504 & 0.442826511887 \\
\hline & $c_{13}$ & -0.604527699539 & -0.154971505863 \\
\hline & $c_{14}$ & 0.118371042255 & 0.036542289696 \\
\hline & $c_{15}$ & 0.008955505675 & 0.006784919880 \\
\hline & $c_{16}$ & 0.219608674529 & 0.401880787461 \\
\hline & $c_{17}$ & 0.017557536680 & 0.006896838546 \\
\hline & $c_{18}$ & 24.442309754388 & 24.751953435615 \\
\hline \multirow[t]{10}{*}{$B_{2}$} & $c_{19}$ & 1.983490330585 & 1.961341923034 \\
\hline & $c_{20}$ & 6.781440647278 & 3.284009890111 \\
\hline & $c_{21}$ & 7.947947040974 & 8.379337236413 \\
\hline & $c_{22}$ & -4.696499003167 & -3.636259490187 \\
\hline & $c_{23}$ & -0.437054238710 & 2.349430558787 \\
\hline & $c_{24}$ & 5.471252046908 & 5.851939658893 \\
\hline & $c_{25}$ & 0.639468224273 & 2.443227221148 \\
\hline & $c_{26}$ & 0.673385731705 & 0.301462797210 \\
\hline & $c_{27}$ & 3.433229044819 & 3.976051353364 \\
\hline & $c_{28}$ & 0.367036215316 & 1.728745711306 \\
\hline
\end{tabular}

CMODH COEFFICIENTS

angle $\theta$, or alternatively, $x=(\theta-40) / 25$. The $B_{0}$ term is ${ }_{276}$ defined as

$$
B_{0}=10^{a_{0}+a_{1} v} f\left(a_{2} v, s_{0}\right)^{\gamma}
$$


where

$$
f\left(s, s_{0}\right)= \begin{cases}\left(s / s_{0}\right)^{\alpha} g\left(s_{0}\right), & s<s_{0} \\ g(s), & s \geq s_{0}\end{cases}
$$

where

$$
g(s)=1 /(1+\exp (-s)) \text { and } \alpha=s_{0}\left(1-g\left(s_{0}\right)\right) .
$$

The functions $a_{0}, a_{1}, a_{2}, \gamma$, and $s_{0}$ depend on incident angle only

$$
\begin{aligned}
a_{0} & =c_{1}+c_{2} x+c_{3} x^{2}+c_{4} x^{3} \\
a_{1} & =c_{5}+c_{6} x \\
a_{2} & =c_{7}+c_{8} x \\
\gamma & =c_{9}+c_{10} x+c_{11} x^{2} \\
s_{0} & =c_{12}+c_{13} x .
\end{aligned}
$$

The $B_{1}$ term is defined as follows:

$$
B_{1}=\frac{c_{14}(1+x)-c_{15} v\left(0.5+x-\tanh \left[4\left(x+c_{16}+c_{17} v\right)\right]\right)}{1+\exp \left(0.34\left(v-c_{18}\right)\right)} \text {. }
$$

The $B_{2}$ term is chosen as

$$
B_{2}=\left(-d_{1}+d_{2} v_{2}\right) \exp \left(-v_{2}\right) .
$$

Here, $v_{2}$ is given by

$$
v_{2}=\left\{\begin{array}{ll}
a+b(y-1)^{n}, & y<y_{0} \\
y, & y \geq y_{0}
\end{array}, y=\frac{v+v_{0}}{v_{0}}\right.
$$

where

$$
\begin{aligned}
y_{0} & =c_{19}, n=c_{20} \\
a & =y_{0}-\left(y_{0}-1\right) / n, b=1 /\left[n\left(y_{0}-1\right)^{n-1}\right] .
\end{aligned}
$$

The quantities $v_{0}, d_{1}$, and $d_{2}$ are functions of incident angle only

$$
\begin{aligned}
& v_{0}=c_{21}+c_{22} x+c_{23} x^{2} \\
& d_{1}=c_{24}+c_{25} x+c_{26} x^{2} \\
& d_{2}=c_{27}+c_{28} x .
\end{aligned}
$$

The coefficients are given in Table III.

\section{REFERENCES}

[1] W. Dierking, "Sea ice monitoring by synthetic aperture radar," Oceanography, vol. 26, no. 2, pp. 100-111, 2013.

[2] G. Spreen, R. Kwok, and D. Menemenlis, "Trends in Arctic sea ice drift and role of wind forcing: 1992-2009," Geophys. Res. Lett., vol. 38, no. 19, Oct. 2011, Art. no. L19501. doi: 10.1029/2011GL048970.

[3] P. R. Holland and R. Kwok, "Wind-driven trends in Antarctic sea-ice drift," Nature Geosci., vol. 5, pp. 872-875, Nov. 2012.

[4] J. T. M. Lenaerts et al., "Meltwater produced by wind-albedo interaction stored in an East Antarctic ice shelf," Nature Climate Change, vol. 7, no. 1, pp. 58-62, Dec. 2016.
[5] A. Stoffelen and D. Anderson, "Scatterometer data interpretation: Estimation and validation of the transfer function CMOD4," J. Geophys. Res., Oceans, vol. 102, no. C3, pp. 5767-5780, Mar. 1997. doi:10.1029/96JC02860.

[6] H. Hersbach, A. Stoffelen, and S. de Haan, "An improved C-band scatterometer ocean geophysical model function: CMOD5," J. Geophys. Res., Oceans, vol. 112, no. C3, Mar. 2007, Art. no. C03006.

[7] H. Hersbach, "Comparison of C-band scatterometer CMOD5.N equivalent neutral winds with ECMWF," J. Atmos. Ocean. Technol., vol. 27, no. 4, pp. 721-736, Apr. 2010.

[8] P. W. Vachon and F. W. Dobson, "Validation of wind vector retrieva from ERS-1 SAR images over the ocean," Global Atmos. Ocean Syst., vol. 5, no. 2, pp. 177-187, 1996

[9] S. Lehner, J. Horstmann, W. Koch, and W. Rosenthal, "Mesoscale wind measurements using recalibrated ERS SAR images," J. Geophys. Res., Oceans, vol. 103, no. C4, pp. 7847-7856, Apr. 1998

[10] J. Horstmann, H. Schiller, J. Schulz-Stellenfleth, and S. Lehner, "Global wind speed retrieval from SAR," IEEE Trans. Geosci. Remote Sens., vol. 41, no. 10, pp. 2277-2286, Oct. 2003.

[11] B. Zhang et al., "Ocean vector winds retrieval from C-band fully polarimetric SAR measurements," IEEE Trans. Geosci. Remote Sens., vol. 50, no. 11, pp. 4252-4261, Nov. 2012.

[12] F. Monaldo, C. Jackson, X. Li, and W. G. Pichel, "Preliminary evaluation of sentinel-1A wind speed retrievals," IEEE J. Sel. Topics Appl. Earth Observ. Remote Sens., vol. 9, no. 6, pp. 2638-2642, Jun. 2016. doi: 10.1109/JSTARS.2015.2504324.

[13] A. Mouche and B. Chapron, "Global C-band Envisat, RADARSAT-2 and Sentinel-1 SAR measurements in copolarization and crosspolarization," J. Geophys. Res., Oceans, vol. 120, no. 11, pp. 7195-7207, Nov. 2015.

[14] Y. Lu, B. Zhang, W. Perrie, A. A. Mouche, X. Li, and H. Wang, "A C-band geophysical model function for determining coastal wind speed using synthetic aperture radar," IEEE J. Sel. Topics Appl. Earth Observ. Remote Sens., vol. 11, no. 7, pp. 2417-2428, Jul. 2018.

[15] D. R. Thompson, T. M. Elfouhaily, and B. Chapron, "Polarization ratio for microwave backscattering from the ocean surface at low to moderate incidence angles," in Proc. IEEE Int. Geosci. Remote Sens. Symp., Los Alamitos, CA, USA, Jul. 1998, pp. 1671-1673. doi: 10.1109/IGARSS.1998.692411.

[16] P. W. Vachon and F. W. Dobson, "Wind retrieval from RADARSAT SAR images: Selection of a suitable C-band $\mathrm{HH}$ polarization wind retrieval model," Can. J. Remote Sens., vol. 26, no. 4, pp. 306-313, Aug. 2000.

[17] J. Horstmann, W. Koch, S. Lehner, and R. Tonboe, "Wind retrieval over the ocean using synthetic aperture radar with C-band $\mathrm{HH}$ polarization," IEEE Trans. Geosci. Remote Sens., vol. 38, no. 5, pp. 2122-2131, Sep. 2000.

[18] A. A. Mouche, D. Hauser, J.-F. Daloze, and C. Guerin, "Dualpolarization measurements at C-band over the ocean: Results from airborne radar observations and comparison with ENVISAT ASAR data," IEEE Trans. Geosci. Remote Sens., vol. 43, no. 4, pp. 753-769, Apr. 2005.

[19] H. Johnsen, G. Engen, and G. Guitton, "Sea-surface polarization ratio from Envisat ASAR AP data," IEEE Trans. Geosci. Remote Sens., vol. 46, no. 11, pp. 3637-3646, Nov. 2008.

[20] B. Zhang, W. Perrie, and Y. He, "Wind speed retrieval from RADARSAT-2 quad-polarization images using a new polarization ratio model," J. Geophys. Res., Oceans, vol. 116, no. C8, Aug. 2011 Art. no. C08008.

[21] G. Liu et al., "A systematic comparison of the effect of polarization ratio models on sea surface wind retrieval from C-band synthetic aperture radar," IEEE J. Sel. Topics Appl. Earth Observ. Remote Sens., vol. 6, no. 3, pp. 1100-1108, Jun. 2013.

[22] A. Stoffelen et al., "Scientific developments and the EPS-SG scatterometer," IEEE J. Sel. Topics Appl. Earth Observ. Remote Sens., vol. 10, no. 5, pp. 2086-2097, May 2017. 


\title{
A Geophysical Model Function for Wind Speed Retrieval From C-Band HH-Polarized Synthetic Aperture Radar
}

\author{
Biao Zhang ${ }^{\circledR}$, Senior Member, IEEE, Alexis Mouche ${ }^{\circledR}$, Yiru Lu, \\ William Perrie $^{(\mathbb{D}}$, Guosheng Zhang ${ }^{(\mathbb{D}}$, and He Wang ${ }^{(\mathbb{1}}$
}

\begin{abstract}
Synthetic aperture radar (SAR) imagery is routinely acquired at $\mathrm{HH}$-polarization in high-latitude areas for measuring surface wind over the ocean. However, in the contrary of VV-polarization, there is no $\mathrm{HH}$-polarization geophysical model function (GMF) exists to directly retrieve wind speed from SAR images. In general, HH-polarized normalized radar cross section (NRCS) is thus converted into VV-polarization and then conventional CMOD functions are used with auxiliary wind direction information for wind speed retrieval. In this letter, we propose a new GMF for SAR ocean surface wind speed retrieval, called CMODH, which relates the C-band NRCS acquired at HH-polarization over the ocean, to the $10-\mathrm{m}$ height wind speed, incident angle, and relative wind direction. We first use more than 220000 ENVISAT ASAR radar backscatter measurements collocated with ASCAT winds to derive the CMODH coefficients. Subsequently, 1459 RADARSAT-2 (RS-2) and 428 Sentinel-1A/B (Sl-1A/B) HH-polarized SAR acquisitions under different wind speeds are matched to in situ buoy observations to validate CMODH. The statistical comparisons between SAR-observed and simulated NRCS show a bias of $-0.07 \mathrm{~dB}$ and a root-mean-square error of $1.62 \mathrm{~dB}$ for RS-2, and $-0.01 \mathrm{~dB}$ and $2.48 \mathrm{~dB}$ for $\mathrm{S1-1A} / \mathrm{B}$. These results suggest that the proposed $\mathrm{CMODH}$ has the potential to directly retrieve ocean surface wind speeds using $\mathrm{C}$-band SAR images acquired at HH-polarization, with no need for NRCS transformation by using various empirical and theoretical polarization ratio models.
\end{abstract}

Index Terms-HH-polarization, ocean surface wind speed, synthetic aperture radar (SAR).

Manuscript received October 21, 2018; revised February 3, 2019; accepted March 13, 2019. This work was supported in part by the National Key Research and Development Program of China under Grant 2016YFC1401001, in part by the National Science Foundation of China for Outstanding Young Scientist under Grant 41622604, in part by the Excellent Youth Science Foundation of Jiangsu Province under Grant BK2016090, in part by the Data Utilization Application Plan of Canadian Space Agency, in part by the Canadian Office of Energy Research and Development, and in part by the ESA Dragon Project. (Corresponding author: Biao Zhang.)

B. Zhang, Y. Lu, and G. Zhang are with the School of Marine Sciences, Nanjing University of Information Science and Technology, Nanjing 210044, China (e-mail: zhangbiao@nuist.edu.cn; lyr@nuist.edu.cn; zgsheng001@gmail.com).

A. Mouche is with IFREMER, Université de Bretagne Occidentale, CNRS, IRD, Laboratoire d'Océanographie Physique et Spatiale (LOPS), 29280 Brest, France (e-mail: alexis.mouche@ifremer.fr).

W. Perrie is with the Bedford Institute of Oceanography, Fisheries and Oceans Canada, Dartmouth, NS B2Y 4A2, Canada (e-mail: william. perrie@dfo-mpo.gc.ca).

H. Wang is with the National Ocean Technology Center, State Oceanic Administration, Tianjin 300112, China.

Color versions of one or more of the figures in this letter are available online at http://ieeexplore.ieee.org.

Digital Object Identifier 10.1109/LGRS.2019.2905578

\section{INTRODUCTION}

$\mathbf{S}$ YNTHETIC aperture radar (SAR) is a very useful microwave sensor for remote sensing of ocean surface features with ability for high-resolution and almost all-weather observations including night and day. Spaceborne SARs are on onboard polar-orbiting satellites and thus have the capability to observe high-latitude areas with shorter revisit time than middle-and low-latitude regions. In particular, they are routinely used to monitor seasonally or annual variations in seaice in the Arctic, Antarctic, and other high-latitude areas such as Chukchi Sea, the Labrador Sea, or the Beaufort Sea. When only one co-polarized channel is available, HH-polarization is preferred for sea-ice application because the contrast with ocean is more pronounced than at VV-polarization [1]. As a consequence, the acquisition strategy at high latitudes gives priority to HH-polarization (and cross-polarization when available). In the contrary, VV is widely at mid-latitude for ocean applications, including wind field measurements.

Moreover, the high-resolution ocean surface wind is not only a key complementary geophysical parameter to investigate sea-ice motion and melt but also the coupling between ocean and the marginal ice zone (MIZ) in high-latitude regions. Long time-series satellite-tracked sea-ice motion observations reveal that changes in wind speed are linked to the observed increase in ice drift speeds in the Center Arctic, on climate timescales [2]. Antarctic sea-ice motions observed at large scale with satellites also suggest that significant trends in Antarctic ice drift can be statistically related to local winds in most geographic areas [3]. For example, in coastal East Antarctica, persistent katabatic winds from the ice sheet's interior cause widespread snow erosion and enhance the melt of blue ice and firn snow [4].

SAR ocean surface wind speed retrieval is usually based on geophysical model functions (GMFs) [5]-[7], which are derived from VV-polarized scatterometer observations. Conventional scatterometer-derived GMFs have been demonstrated to be applicable for moderate wind speed retrieval with an accuracy of $1.5 \mathrm{~m} / \mathrm{s}$ from ERS, ENVISAT ASAR, Sentinel-1A, or RADARSAT-2 VV-polarized SAR images [8]-[12]. Mouche and Chapron [13] pioneered the idea of relying directly and massively on SAR data to describe the relationship between NRCS and ocean surface wind in both co-polarizations and possibly derive GMF from SAR data. 
Recently, a new GMF has been proposed for C-band SAR coastal wind speed mapping [14], by using SAR measurements acquired in VV-polarization collocated with buoy observations. However, no similar well-documented wind retrieval model exists to derive wind speed from the HH-polarized SAR imagery. Therefore, various empirical and theoretical polarization ratio (PR) models have been developed to transform normalized radar cross section (NRCS) from $\mathrm{HH}$ into VV-polarization [15]-[20], along with CMOD GMFs to retrieve wind speed. A systematic comparison of PR models for ocean surface wind retrieval using ENVISAT ASAR images shows a small wind speed underestimation or overestimation at very small or large incident angles, respectively [21].

Although the CMOD GMF-PR approach constitutes a practical hybrid model for wind speed retrieval from SAR images acquired at HH-polarization, the NRCS transformation certainly induces error in wind speed estimation. In order to directly retrieve wind speeds using $\mathrm{HH}$-polarized SAR imagery, we develop a new GMF relating NRCS at $\mathrm{HH}$-polarization to radar incident angle, wind speed at $10 \mathrm{~m}$ height, and relative wind direction. Finally, to complement the VV-polarization, the next European scatterometer mission onboard MetOp-SG will also operate at HH-polarization (and VH-polarization) [22]. The proposed GMF should, thus, directly benefit to this forthcoming satellite mission. This letter is organized as follows. Data sets are described in Section II. GMF development and validation are presented in Section III. Summary and conclusion are given in Section IV.

\section{DATA SET}

In this letter, more than 2700 ENVISAT/ASAR images acquired in wide swath mode (WSM) in 2009 are collocated with MetOp/ASCAT and ECMWF winds to develop the HH-polarized GMF. The incident angles of WSM are between $16^{\circ}$ and $42^{\circ}$ in the near and far ranges, respectively. SAR acquisitions in WSM cover about $400 \mathrm{~km}$ in the range direction and can be more than $1000 \mathrm{~km}$ in the azimuth direction. We processed all ASAR WSM products to derive the NRCS, incident angles, and azimuth angles at a spatial resolution cell of $12.5 \mathrm{~km}$. These measurements are then matched to ASCAT wind speeds and directions. This results in more than 220000 collocations. The ENVISAT ASAR and ASCAT orbits and field of view enable temporal intervals of less than $1 \mathrm{~h}$, for collocation. This collocated data set has already been used to analyze wind sensitivity for different incident angles and azimuth angles [13].

In order to validate the proposed GMF, we collect 1352 RS-2 SAR images acquired in quad-polarization $(\mathrm{HH}+\mathrm{HV}+$ $\mathrm{VH}+\mathrm{VV})$ and 107 dual-polarization $(\mathrm{HH}+\mathrm{HV})$ imaging modes between October 2008 and April 2013, and 428 Sentinel-1A/B (Sl-1A/B) images acquired in extra wide swath (EW) mode between October 2014 and April 2018. A summary of the parameters for the RS-2 quad-and dualpolarization modes, including incident angles, spatial resolutions, swaths, and noise-equivalent sigma-zero (NESZ) values, is given in Table I. The EW mode of S1-1A/B data has the capability to observe the ocean surface with dual-polarization $(\mathrm{HH}+\mathrm{HV})$ channels. Table II contains the major parameters
TABLE I

MAJOR PARAMETERS OF THE RS-2 QUAD-AND DUAL-POLARIZATION IMAGING MODES

\begin{tabular}{lcc}
\hline Parameter & quad-polarization mode & $\begin{array}{c}\text { dual-polarization } \\
\text { ScanSAR Narrow mode }\end{array}$ \\
\hline Polarization & $\mathrm{HH}+\mathrm{HV}+\mathrm{VH}+\mathrm{VV}$ & $\mathrm{VV}+\mathrm{VH}$ \\
Incident angles & $20^{\circ}-49^{\circ}$ & $20^{\circ}-46^{\circ}$ \\
Azimuth resolution & $8 \mathrm{~m}$ & $60 \mathrm{~m}$ \\
Range resolution & $5.4 \mathrm{~m}$ & $79.9-37.7 \mathrm{~m}$ \\
Swath & $25 \mathrm{~km}$ & $300 \mathrm{~km}$ \\
NESZ & $-36.5 \pm 3 \mathrm{~dB}$ & $28.5 \pm 2.5 \mathrm{~dB}$ \\
\hline
\end{tabular}

TABLE II

Major Parameters of THE SL-1A/B IW AND EW IMAgIng ModeS

\begin{tabular}{lc}
\hline Parameter & Extra wide swath (EW) arrow mode \\
\hline Polarization & $\mathrm{HH}+\mathrm{HV}$ \\
Incident angles & $19^{\circ}-47^{\circ}$ \\
Azimuth resolution & $40 \mathrm{~m}$ \\
Range resolution & $20 \mathrm{~m}$ \\
Swath & $400 \mathrm{~km}$ \\
NESZ & $-22 \mathrm{~dB}$ \\
\hline
\end{tabular}

for EW mode. All RS-2 and Sl-1A/B SAR images are collocated with 102 in situ National Data Buoy Center (NDBC) buoys in the Gulf of Alaska, off the East and West coasts of the USA, and the European Seas. The time interval for the collocation is smaller than $30 \mathrm{~min}$. We process the NRCS at $\mathrm{HH}$-polarization at a spatial resolution of $10 \mathrm{~km}$ to match the resolution used by Mouche and Chapron [13], where medium (instead of high) resolution was used to be in line with collocated wind field from ASCAT. This approach results in 1527 and 461 collocated data pairs for RS-2 and Sl-1A/B, respectively, which are used to compare simulated and SAR-measured NRCS.

\section{Model Development and Validation}

Similar to NRCS at VV-polarization, HH-polarized NRCS also depend on radar incident angle, wind speed, and direction (see [13, Fig. 3(b) and (d)]). Therefore, we describe NRCS at HH-polarization by a nonlinear mapping function of the incident angle, wind speed, and relative wind direction (the angle between the true wind direction and the radar observation direction), which is given as

$$
\begin{aligned}
\sigma_{0}^{\mathrm{hh}}(v, \phi, \theta)=\left(B_{0}(v, \theta)[1+\right. & B_{1}(v, \theta) \cos (\phi) \\
& \left.\left.+B_{2}(v, \theta) \cos (2 \phi)\right]\right)^{p}
\end{aligned}
$$

where the isotropic $B_{0}$, the upwind/downwind $B_{1}$, and the upwind/crosswind $B_{2}$ amplitude terms are the functions of wind speed $v$ and incident angle $\theta$. The superscript $p$ is a constant with a value of 1.6. The superscript $p$ and the transfer functions used to define $B_{0}, B_{1}$, and $B_{2}$ are adopted from CMOD5 [6] for use in this study. The proposed form of the GMF is almost the same as that of CMOD5 excepted for the $p$ exponent, on $B_{0}$.

For each incident angle and wind speed bin, using the collocated ENVISAT ASAR and ASCAT data set, we first analyze the NRCS at HH-polarization as a function of azimuth angle 


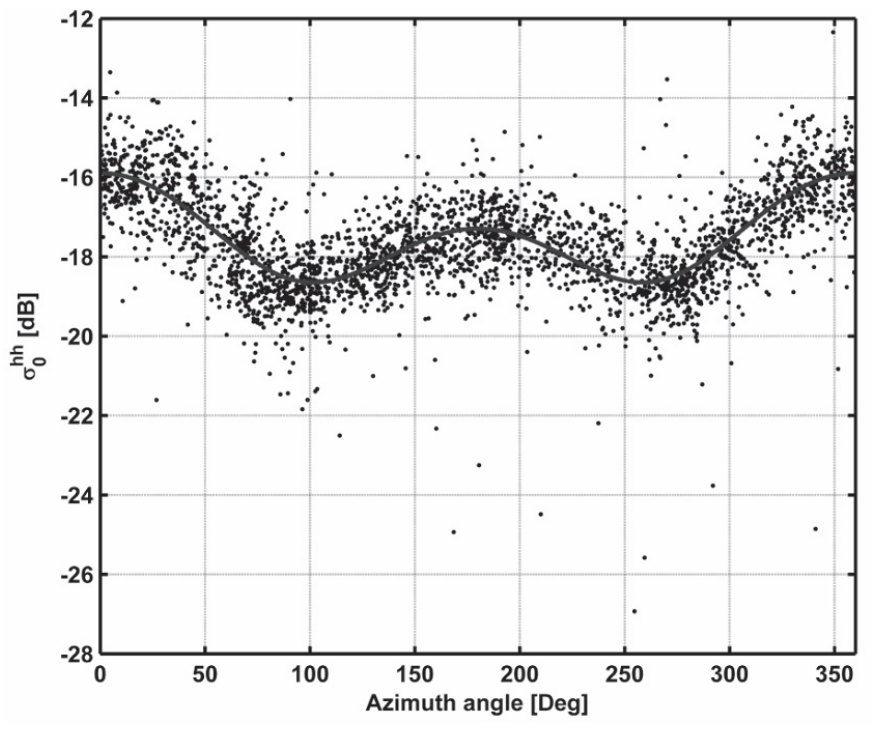

Fig. 1. HH-polarized NRCS as a function of azimuth angle, for a $7 \mathrm{~m} / \mathrm{s}$ wind speed and $35^{\circ}$ incidence angle. Black dots are SAR-observed NRCS at $\mathrm{HH}$-polarization. Blue line represents regression results.
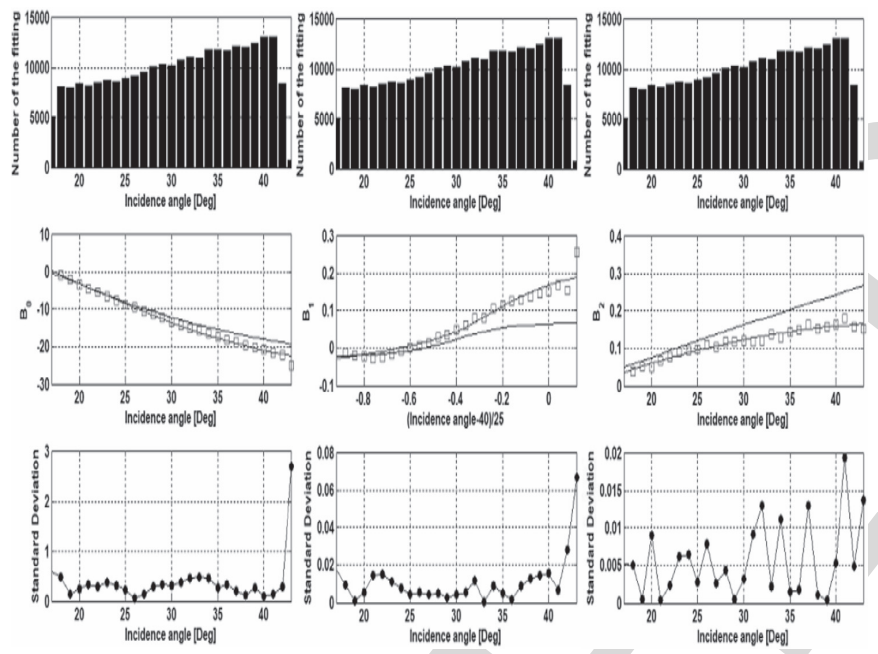

Fig. 2. Number of the data for (Top) $B_{i}$ coefficients derivation, (Middle) $B_{i}$ coefficients, and (Bottom) standard deviation of the regression as a function of incident angle for $7 \mathrm{~m} / \mathrm{s}$ wind speed. $B_{i}$ coefficients derived from (1), the regression analysis approach, and the CMOD5 are represent as red squares, and blue and black solid lines, respectively.

to determine the corresponding $B_{i}(i=0,1,2)$ coefficients by using a regression analysis approach. Fig. 1 presents the ASAR-observed NRCS (black dots) versus azimuth angles for $7 \mathrm{~m} / \mathrm{s}$ wind speed and $35^{\circ}$ incident angle. Fig. 1 clearly shows the azimuth modulation of NRCS at HH-polarization. Estimated NRCS values using (1) are indicated in the blue solid line in Fig. 1, which are in good agreement with the radar observations, suggesting that (1) is appropriate to describe the proposed GMF.

Subsequently, to further assess the rationality of the functional form, $B_{i}$ coefficients are also analyzed as a function of wind speed and incident angle. The top three panels in Fig. 2 show the number of data used for $B_{i}$ coefficients derivation. For $7 \mathrm{~m} / \mathrm{s}$ wind speed and different incident angles, $B_{i}$ coefficients derived from (1), the regression analysis

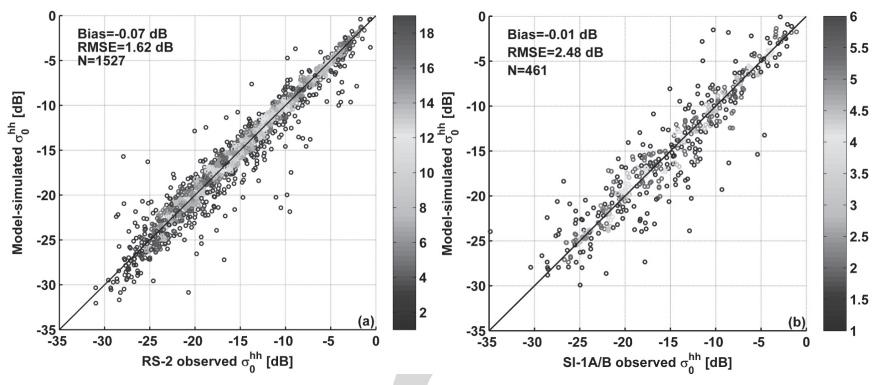

Fig. 3. Comparisons of observed NRCS in HH-polarization ( $x$-axis) from (a) RS-2 and (b) S1-A/B with CMODH model simulations ( $y$-axis). The color bar indicates the scatter density.

approach, and the CMOD5 function are represented as red squares, and blue and black solid lines, in the middle three panels of Fig. 2.

Overall, the trend of $B_{i}$ coefficients obtained by our analysis and those provided by CMOD5 GMF are in very good agreement excepted at the largest incident angle of $43^{\circ}$. The $B_{0}$ values from the regression analysis and from CMOD5 estimation are almost identical at low incident angles $\left(<23^{\circ}\right)$. As incident angles increase, the discrepancies between $B_{i}$ coefficients obtained for $\mathrm{HH}$ from our regression analysis and from CMOD5 gradually increase. This is in line with a known different sensitivity to ocean sea-surface backscattering between VV- and HH-polarizations. Indeed, at C-band, polarization effect is strongly incident angle-dependent. In particular, our analysis confirms that $B_{1}$ values at $\mathrm{VV}$ are smaller than at $\mathrm{HH}$, while $B_{0}$ and $B_{2}$, are larger at VV.

The standard deviations of the regression analysis for each incident angle bin are illustrated in the bottom three panels in Fig. 2. The standard deviation for the large incident angle bin of $43^{\circ}$ is much larger than those for other incident angle bins, which is possibly caused by the very few data numbers in this bin. Consequently, we do not use NRCS for this large incident angle bin to develop our $\mathrm{HH}$-polarized GMF. In this study, $B_{i}$ coefficients are determined via a nonlinear weighted regression approach by minimizing the standard deviation between ENVISAT ASAR observations and regression analysis for all wind speeds and incident angle bins. The number of the data in each wind speed and incident angle bin is considered as a weighted factor for the regression analysis. The final model formulation and 28 coefficients for HH-polarization are given in the Appendix. Since our collocated data sets both involve NRCS at HH- and VVpolarization, we also use (1) to derive the coefficients of GMF for VV-polarization, which are shown in the Appendix. Therefore, one can estimate NRCS at HH-and VV-polarization using (1) and coefficients with given incident angle, wind speed, and direction.

To assess the accuracy of the proposed GMF, we make statistical validations using collocated RS-2 and Sl-1A/B observations and buoy measurements. Fig. 3(a) shows the simulated and SAR-measured NRCS at HH-polarization, with a bias of $-0.07 \mathrm{~dB}$ and a RMSE of $1.62 \mathrm{~dB}$. For comparison between model simulations and S1-A/B observations, the bias and RMSE are -0.01 and $2.48 \mathrm{~dB}$, which are shown in Fig. 3(b). For NRCS comparisons, the RS-2 data have smaller 
RMSE than the S1-1A/B data. The reason for this result may be associated with two factors: 1) RS-2 has lower NESZ than the $\mathrm{S} 1-1 \mathrm{~A} / \mathrm{B}$ and 2) the collocations between $\mathrm{S} 1-1 \mathrm{~A} / \mathrm{B}$ and buoy data are less accurate than that of RS-2. For S1-1A/B collocation, some buoys are outside the SAR image which leads to the large spatial interval of the collocation. However, for RS-2 collocation, each buoy locates in the SAR image and thus there is almost no spatial match error.

\section{CONCLUSION}

Accurate wind field information in high latitudes and polar regions plays an important role in sea-ice motion and melt simulations and predictions. The high sensitivity of SAR backscatter at HH-polarization to sea-ice makes it more preferable for sea-ice monitoring, rather than $\mathrm{VV}$-polarization. However, unlike CMOD GMFs, no HH-polarized GMF can be directly used to retrieve ocean surface wind speed. As a result, various empirical and theoretical PR models are used to transform NRCS from HH into VV-polarization, which induces inevitable errors in the retrieved wind speeds. In order to solve this problem, we develop a new HH-polarized GMF for C-band SAR, taking advantage of a large number of C-band SAR data and collocated scatterometer wind measurements. The proposed GMF has potential to improve SAR wind speed retrieval accuracy and can be used to directly retrieve wind speed in mixing areas of ice and water, like the MIZ.

The new GMF relates NRCS at HH-polarization to radar incident angle, wind speed at $10 \mathrm{~m}$ height, and relative wind direction. We use a nonlinear weighted regression method and the collocated data set (ENVISAT ASAR backscatter and ASCAT winds) to derive all the coefficients in the GMF. Furthermore, we use an independent matching of data (RS-2 and S1-1A/B backscatter and buoy measurements) to validate the proposed GMF. The statistical comparisons between radar observations and GMF simulations show a bias of $-0.07 \mathrm{~dB}$ and a root-mean-square error of $1.62 \mathrm{~dB}$ for RS-2, and $-0.01 \mathrm{~dB}$ and $2.48 \mathrm{~dB}$ for S1-1A/B, respectively. Based on this new GMF, we do not need to make a NRCS transformation with a PR model before wind speed retrieval. The scatterometer (SCA) onboard EUMETSAT Polar System-Second Generation (EPS-SG) mission shall provide observations with VV-, HH-, or VH-polarization. The addition of HH- or VH-polarization is considered as an option for extending the upper limit of the dynamic measurement range. Therefore, the proposed GMF can be a benefit for EPS-SG SCA wind retrieval. Moreover, this also opens new perspectives for the forthcoming launch of the RADARSAT Constellation Mission (RCM) with new polarization configurations.

\section{APPENDIX \\ CMODH MODEL FORMULATION AND COEFFICIENTS}

The form of the CMODH model is

$$
\begin{aligned}
\sigma_{0}^{\mathrm{hh}}(v, \phi, \theta)=\left(B_{0}(v, \theta)[1\right. & +B_{1}(v, \theta) \cos (\phi) \\
& \left.\left.+B_{2}(v, \theta) \cos (2 \phi)\right]\right)^{p}
\end{aligned}
$$

where $p$ is constant with a value of $1.6, B_{0}, B_{1}$, and $B_{2}$ are functions of wind speeds $v$ and the incidence
TABLE III

\begin{tabular}{|c|c|c|c|}
\hline Function & Coefficients & $\mathrm{HH}$ & VV \\
\hline \multirow[t]{18}{*}{$B_{0}$} & $c_{1}$ & -0.72722756511 & -0.13393789593 \\
\hline & $c_{2}$ & -1.1901195406 & -0.74081314533 \\
\hline & $c_{3}$ & 0.33968637656 & 0.34811480603 \\
\hline & $c_{4}$ & 0.086759069544 & 0.019382338942 \\
\hline & $c_{5}$ & 0.003090124916 & -0.008066293463 \\
\hline & $c_{6}$ & 0.011761378188 & 0.006426074015 \\
\hline & $c_{7}$ & 0.129158495658 & 0.096343783534 \\
\hline & $c_{8}$ & 0.083506931034 & 0.042280179737 \\
\hline & $c_{9}$ & 4.092557781322 & 5.007750349297 \\
\hline & $c_{10}$ & 1.211169044551 & 0.717396068916 \\
\hline & $c_{11}$ & -1.119776245438 & -1.501296438845 \\
\hline & $c_{12}$ & 0.579066509504 & 0.442826511887 \\
\hline & $c_{13}$ & -0.604527699539 & -0.154971505863 \\
\hline & $c_{14}$ & 0.118371042255 & 0.036542289696 \\
\hline & $c_{15}$ & 0.008955505675 & 0.006784919880 \\
\hline & $c_{16}$ & 0.219608674529 & 0.401880787461 \\
\hline & $c_{17}$ & 0.017557536680 & 0.006896838546 \\
\hline & $c_{18}$ & 24.442309754388 & 24.751953435615 \\
\hline \multirow[t]{10}{*}{$B_{2}$} & $c_{19}$ & 1.983490330585 & 1.961341923034 \\
\hline & $c_{20}$ & 6.781440647278 & 3.284009890111 \\
\hline & $c_{21}$ & 7.947947040974 & 8.379337236413 \\
\hline & $c_{22}$ & -4.696499003167 & -3.636259490187 \\
\hline & $c_{23}$ & -0.437054238710 & 2.349430558787 \\
\hline & $c_{24}$ & 5.471252046908 & 5.851939658893 \\
\hline & $c_{25}$ & 0.639468224273 & 2.443227221148 \\
\hline & $c_{26}$ & 0.673385731705 & 0.301462797210 \\
\hline & $c_{27}$ & 3.433229044819 & 3.976051353364 \\
\hline & $c_{28}$ & 0.367036215316 & 1.728745711306 \\
\hline
\end{tabular}

CMODH COEFFICIENTS

angle $\theta$, or alternatively, $x=(\theta-40) / 25$. The $B_{0}$ term is ${ }_{276}$ defined as

$$
B_{0}=10^{a_{0}+a_{1} v} f\left(a_{2} v, s_{0}\right)^{\gamma}
$$


where

$$
f\left(s, s_{0}\right)= \begin{cases}\left(s / s_{0}\right)^{\alpha} g\left(s_{0}\right), & s<s_{0} \\ g(s), & s \geq s_{0}\end{cases}
$$

where

$$
g(s)=1 /(1+\exp (-s)) \text { and } \alpha=s_{0}\left(1-g\left(s_{0}\right)\right) .
$$

The functions $a_{0}, a_{1}, a_{2}, \gamma$, and $s_{0}$ depend on incident angle only

$$
\begin{aligned}
a_{0} & =c_{1}+c_{2} x+c_{3} x^{2}+c_{4} x^{3} \\
a_{1} & =c_{5}+c_{6} x \\
a_{2} & =c_{7}+c_{8} x \\
\gamma & =c_{9}+c_{10} x+c_{11} x^{2} \\
s_{0} & =c_{12}+c_{13} x .
\end{aligned}
$$

The $B_{1}$ term is defined as follows:

$$
B_{1}=\frac{c_{14}(1+x)-c_{15} v\left(0.5+x-\tanh \left[4\left(x+c_{16}+c_{17} v\right)\right]\right)}{1+\exp \left(0.34\left(v-c_{18}\right)\right)} \text {. }
$$

The $B_{2}$ term is chosen as

$$
B_{2}=\left(-d_{1}+d_{2} v_{2}\right) \exp \left(-v_{2}\right) .
$$

Here, $v_{2}$ is given by

$$
v_{2}=\left\{\begin{array}{ll}
a+b(y-1)^{n}, & y<y_{0} \\
y, & y \geq y_{0}
\end{array}, y=\frac{v+v_{0}}{v_{0}}\right.
$$

where

$$
\begin{aligned}
y_{0} & =c_{19}, n=c_{20} \\
a & =y_{0}-\left(y_{0}-1\right) / n, b=1 /\left[n\left(y_{0}-1\right)^{n-1}\right] .
\end{aligned}
$$

The quantities $v_{0}, d_{1}$, and $d_{2}$ are functions of incident angle only

$$
\begin{aligned}
& v_{0}=c_{21}+c_{22} x+c_{23} x^{2} \\
& d_{1}=c_{24}+c_{25} x+c_{26} x^{2} \\
& d_{2}=c_{27}+c_{28} x .
\end{aligned}
$$

The coefficients are given in Table III.

\section{REFERENCES}

[1] W. Dierking, "Sea ice monitoring by synthetic aperture radar," Oceanography, vol. 26, no. 2, pp. 100-111, 2013.

[2] G. Spreen, R. Kwok, and D. Menemenlis, "Trends in Arctic sea ice drift and role of wind forcing: 1992-2009," Geophys. Res. Lett., vol. 38, no. 19, Oct. 2011, Art. no. L19501. doi: 10.1029/2011GL048970.

[3] P. R. Holland and R. Kwok, "Wind-driven trends in Antarctic sea-ice drift," Nature Geosci., vol. 5, pp. 872-875, Nov. 2012.

[4] J. T. M. Lenaerts et al., "Meltwater produced by wind-albedo interaction stored in an East Antarctic ice shelf," Nature Climate Change, vol. 7, no. 1, pp. 58-62, Dec. 2016.
[5] A. Stoffelen and D. Anderson, "Scatterometer data interpretation: Estimation and validation of the transfer function CMOD4," J. Geophys. Res., Oceans, vol. 102, no. C3, pp. 5767-5780, Mar. 1997. doi:10.1029/96JC02860.

[6] H. Hersbach, A. Stoffelen, and S. de Haan, "An improved C-band scatterometer ocean geophysical model function: CMOD5," J. Geophys. Res., Oceans, vol. 112, no. C3, Mar. 2007, Art. no. C03006.

[7] H. Hersbach, "Comparison of C-band scatterometer CMOD5.N equivalent neutral winds with ECMWF," J. Atmos. Ocean. Technol., vol. 27, no. 4, pp. 721-736, Apr. 2010.

[8] P. W. Vachon and F. W. Dobson, "Validation of wind vector retrieva from ERS-1 SAR images over the ocean," Global Atmos. Ocean Syst., vol. 5, no. 2, pp. 177-187, 1996

[9] S. Lehner, J. Horstmann, W. Koch, and W. Rosenthal, "Mesoscale wind measurements using recalibrated ERS SAR images," J. Geophys. Res., Oceans, vol. 103, no. C4, pp. 7847-7856, Apr. 1998.

[10] J. Horstmann, H. Schiller, J. Schulz-Stellenfleth, and S. Lehner, "Globa wind speed retrieval from SAR," IEEE Trans. Geosci. Remote Sens., vol. 41, no. 10, pp. 2277-2286, Oct. 2003.

[11] B. Zhang et al., "Ocean vector winds retrieval from C-band fully polarimetric SAR measurements," IEEE Trans. Geosci. Remote Sens., vol. 50, no. 11, pp. 4252-4261, Nov. 2012.

[12] F. Monaldo, C. Jackson, X. Li, and W. G. Pichel, "Preliminary evaluation of sentinel-1A wind speed retrievals," IEEE J. Sel. Topics Appl. Earth Observ. Remote Sens., vol. 9, no. 6, pp. 2638-2642, Jun. 2016. doi: 10.1109/JSTARS.2015.2504324.

[13] A. Mouche and B. Chapron, "Global C-band Envisat, RADARSAT-2 and Sentinel-1 SAR measurements in copolarization and crosspolarization," J. Geophys. Res., Oceans, vol. 120, no. 11, pp. 7195-7207, Nov. 2015.

[14] Y. Lu, B. Zhang, W. Perrie, A. A. Mouche, X. Li, and H. Wang, "A C-band geophysical model function for determining coastal wind speed using synthetic aperture radar," IEEE J. Sel. Topics Appl. Earth Observ. Remote Sens., vol. 11, no. 7, pp. 2417-2428, Jul. 2018.

[15] D. R. Thompson, T. M. Elfouhaily, and B. Chapron, "Polarization ratio for microwave backscattering from the ocean surface at low to moderate incidence angles," in Proc. IEEE Int. Geosci. Remote Sens. Symp., Los Alamitos, CA, USA, Jul. 1998, pp. 1671-1673. doi: 10.1109/IGARSS.1998.692411.

[16] P. W. Vachon and F. W. Dobson, "Wind retrieval from RADARSAT SAR images: Selection of a suitable C-band $\mathrm{HH}$ polarization wind retrieval model," Can. J. Remote Sens., vol. 26, no. 4, pp. 306-313, Aug. 2000.

[17] J. Horstmann, W. Koch, S. Lehner, and R. Tonboe, "Wind retrieval over the ocean using synthetic aperture radar with C-band $\mathrm{HH}$ polarization," IEEE Trans. Geosci. Remote Sens., vol. 38, no. 5, pp. 2122-2131, Sep. 2000.

[18] A. A. Mouche, D. Hauser, J.-F. Daloze, and C. Guerin, "Dualpolarization measurements at C-band over the ocean: Results from airborne radar observations and comparison with ENVISAT ASAR data," IEEE Trans. Geosci. Remote Sens., vol. 43, no. 4, pp. 753-769, Apr. 2005.

[19] H. Johnsen, G. Engen, and G. Guitton, "Sea-surface polarization ratio from Envisat ASAR AP data," IEEE Trans. Geosci. Remote Sens., vol. 46, no. 11, pp. 3637-3646, Nov. 2008.

[20] B. Zhang, W. Perrie, and Y. He, "Wind speed retrieval from RADARSAT-2 quad-polarization images using a new polarization ratio model," J. Geophys. Res., Oceans, vol. 116, no. C8, Aug. 2011 Art. no. C08008.

[21] G. Liu et al., "A systematic comparison of the effect of polarization ratio models on sea surface wind retrieval from C-band synthetic aperture radar," IEEE J. Sel. Topics Appl. Earth Observ. Remote Sens., vol. 6, no. 3, pp. 1100-1108, Jun. 2013.

[22] A. Stoffelen et al., "Scientific developments and the EPS-SG scatterometer," IEEE J. Sel. Topics Appl. Earth Observ. Remote Sens., vol. 10, no. 5, pp. 2086-2097, May 2017. 